\title{
Long non-coding RNA SNHG6 promotes tumorigenesis of nasopharyngeal carcinoma by competitively binding to miR-944 with RGS17
}

\section{Yu'e Han}

Qilu Hospital of Shandong University (Qingdao)

\section{Xing Liu}

Qingdao Hospital of Traditional Chinese Medicine

\section{Guangling Li}

Qingdao Eighth People's Hospital

Xia Ju

Qingdao Jimo People's Hospital

Zhongyi Song ( $\sim$ lay666622@163.com )

Qilu Hospital of Shandong University https://orcid.org/0000-0002-2338-6618

\section{Research}

Keywords: SNHG6, nasopharyngeal carcinoma, miR-944, RGS17

Posted Date: May 1st, 2020

DOl: https://doi.org/10.21203/rs.3.rs-25032/v1

License: (9) This work is licensed under a Creative Commons Attribution 4.0 International License. Read Full License 


\section{Abstract \\ Background}

Previous studies have shown that many long noncoding RNAs (IncRNAs) are involved in the pathogenesis of nasopharyngeal carcinoma (NPC). However, the regulatory mechanism of IncRNA SNHG6 remains unknown. Therefore, this study was design to preliminarily elucidate the role of SNHG6 in NPC.

\section{Methods}

The mRNA expression was detected by RT-qPCR. CCK-8, Transwell and dual luciferase reporter assays were used to investigate the function of SNHG6 in NPC.

\section{Results}

Upregulation of SNHG6 and downregulation of miR-944 were identified in NPC and were associated with TNM stage and distant metastasis in NPC patients. Additionally, SNHG6 acts as a molecular sponge of miR-944. More importantly, SNHG6 promoted NPC cell proliferation, migration and invasion by downregulating miR-944. Further, RGS17 was confirmed to be a direct target of miR-944. MiR-944 restrained NPC progression by targeting RGS17. Besides that, knockdown of RGS17 was found to block NPC progression. Upregulation of SNHG6 weakened the suppressive effect of RGS17 knockdown in NPC.

\section{Conclusion}

LncRNA SNHG6 promotes tumorigenesis of NPC by competitively binding to miR-944 with RGS17.

\section{Introduction}

Nasopharyngeal carcinoma (NPC) is a malignant tumor that occurs on the top and side walls of the nasopharyngeal cavity. The preferred treatment for NPC patients is radiation therapy [1]. NPC has a special ethnic aggregation and family heredity, so it still maintains a high incidence in some areas [2]. Moreover, the location of NPC is more concealed, and early symptoms are not obvious. Thus, NPC is easy to be missed and misdiagnosed [3]. At present, early treatment and early detection have a good prognosis for NPC. Therefore, there is an urgent need to develop new molecular markers for the diagnosis and treatment of NPC.

With the continuous development of high-throughput gene sequencing technology, a large number of non-coding RNAs (ncRNAs) have been discovered. Today, more and more literature proves that ncRNA can participate in a variety of biological and disease processes [4]. As a type of ncRNA, IncRNA has been 
widely investigated in human cancers including NPC. For example, IncRNA NEAT1 targeted miR-34a-5p and derived NPC progression [5]. On the contrary, IncRNA LINC01133 expression was decreased in NPC and restrained NPC tumorigenesis by binding to YBX1 [6]. The role of small nucleolar RNA host gene 6 (SNHG6) has been extensively investigated in human malignancy. Upregulation of SNHG6 has been detected in many human cancers, such as breast cancer and colorectal cancer $[7,8]$. Functionally, IncRNA SNHG6 acted as a prognostic factor to regulate cell proliferation in glioma [9]. In addition, upregulation of IncRNA SNHG6 was found to promote esophageal squamous cell carcinoma cell malignancy and its diagnostic value [10]. However, the function of SNHG6 in NPC remains unclear. Therefore, this research was designed to solve the above problem.

Previous studies have demonstrated that IncRNA functions as a ceRNA for miRNAs to regulate tumorigenesis. LncRNA SNHG6 has been found to promote cell proliferation and EMT through sponging miR-101-3p [11]. Here, miR-944 was predicted to be an underlying target of IncRNA SNHG6. The abnormal expression of miR-944 has been identified in human cancers. Downregulation of miR-944 has been found in non-small cell lung cancer and colorectal cancer $[12,13]$. But miR-944 was upregulated in endometrial cancer and breast cancer $[14,15]$. These findings indicate that the expression level of miR-944 depends on the type of cancers. However, the function of miR-944 as well as its relationship with in IncRNA SNHG6 in NPC is largely unknown and need to be explored.

It has been reported that IncRNA SNHG6 regulates EZH2 expression by sponging miR-214 in colorectal cancer [16]. Therefore, we suspect that IncRNA SNHG6 may regulate the target gene of miR-944 in NPC. In this study, Regulator of G Protein Signaling 17 (RGS17) was found to have a binding site with miR-944, which has not been reported in previous studies. Li et al has reported that RGS17 was upregulated and promoted tumor growth and migration in [17]. In addition, Hsa-miR-182 suppressed lung tumorigenesis through downregulation of RGS17 expression in vitro [18]. But the interaction between RGS17 and SNHG6/miR-944 axis has not been investigated in previous studies. Therefore, we investigate the regulatory mechanism of SNHG6/miR-944/RGS17 in NPC. This study will help us better understand the pathogenesis of NPC.

\section{Materials And Methods}

\section{Clinical tissues}

This study was approved by the Institutional Ethics Committee of Qilu Hospital of Shandong University. The experimental samples were obtained from 56 patients with NPC in Qilu Hospital of Shandong University. All patients provided written informed consents. These tissues were frozen in liquid nitrogen and stored at $-80^{\circ} \mathrm{C}$.

\section{Cell lines and culture}

Normal nasopharyngeal epithelial cell line NP69 and C666-1 NPC cells were purchased from BeNa Culture Collection (BNCC, Beijing, China). DMEM medium with 10\% FBS (Gibco, Grand Island, NY, USA) 
was used to incubate these cells in an atmosphere with $5 \% \mathrm{CO}_{2}$ at $37^{\circ} \mathrm{C}$.

\section{Cell transfection}

The pcDNA3.1 vector with SNHG6 and RGS17 complementary DNA, SNHG6 and RGS17 siRNA or miR944 mimics and inhibitor were obtained from Ribobio (Guangzhou, China). Next, Lipofectamine 2000 (Invitrogen/Thermo Fisher Scientifc) was used to transfect them in C666-1 cells.

\section{RT-qPCR}

TRIzol reagent (Invitrogen, USA) was used to extract total RNA. RNA was reversely transcript into complementary DNA (cDNA) using PrimeScript RT reagent kit (Takara, Dalian, China). SYBR Premix Ex Taq GC Kit (Thermo Fisher Scientific) and primers were used to perform RT-qPCR assay. U6 or GAPDH was used as an internal reference for RNA and gene. Their expressions were quantified with the $2^{-} \triangle \triangle \mathrm{cq}$ method.

\section{Cell Counting Kit-8 (CCK-8) assay}

Transfected C666- 1 cells ( $4 \times 10^{3}$ cells/well) in a 96-well plate were incubated for $24,48,72$ or $96 \mathrm{~h}$, respectively. Then, the cells were incubated with $10 \mu \mathrm{L}$ CCK-8 reagents for $4 \mathrm{~h}$. The medium was discarded and dimethyl sulfoxide was added. The absorbance at $450 \mathrm{~nm}$ was detected by a microplate reader (Olympus Corp., Tokyo, Japan).

\section{Transwell assay}

Cell migration and invasion were detected in the upper chamber without or with Matrigel. Then, transfected C666-1 cells ( $4 \times 10^{3}$ cells/well) were placed in Transwell upper chamber. Lower chamber was added with DMEM medium with $10 \%$ FBS. After $24 \mathrm{~h}$ of incubation, the moved cells were stained with $0.1 \%$ crystal violet. Observation and photographing were performed by a light microscope.

\section{Dual luciferase reporter assay}

The pmiR-GLO vector with wt-SNHG6 and mut-SNHG6 or wt-RGS17 and mut-RGS17 was synthesized by Promega Beijing Biotech (Beijing, China). Next, C666-1 cells with the above reporter plasmids and miR944 mimics were incubated for $48 \mathrm{~h}$. Firefly and Renilla luciferase activities were determined by dualluciferase reporter assay system (Promega, USA).

\section{Statistical analysis}

Data are shown as was mean \pm SD. It was analyzed by SPSS 19.0 or Graphpad Prism 6. Differences were analyzed using Student's t-test or one-way ANOVA. $P<0.05$ was regarded as statistical significance.

\section{Results}




\section{The abnormal expression of SNHG6 and miR-944 in NPC}

Here, the abnormal expression of SNHG6 and miR-944 was detected examined in NPC. SNHG6 expression was increased in NPC tissues compared with normal tissues (Fig. 1A). Additionally, SNHG6 was upregulated in stage III/IV and distant metastasis tissues (Fig. 1B, 1C). It indicates that SNHG6 expression may be related to TNM stage and distant metastasis in NPC patients. Meanwhile, upregulation of SNHG6 was found in C666-1 NPC cells contrast to normal nasopharyngeal epithelial cell NP69 (Fig. 1D). Next, miR-944 was found to be downregulated in NPC tissues compared to normal tissues (Fig. 1E). Furthermore, low miR-944 expression was measured in stage III/IV and distant metastasis tissues (Fig. 1F, 1G), suggesting that miR-944 expression may be related to TNM stage and distant metastasis in NPC patients. Decreased expression of miR-944 was also detected in C666-1 cells compared to NP69 cells (Fig. 1H). Based on these results, we suspect that SNHG6 and miR-944 may be involved in the pathogenesis of NPC.

\section{SNHG6 acts as a molecular sponge of miR-944.}

Next, SNHG6 was found to have a binding site with SNHG6 in starBase database (http://starbase.sysu.edu.cn/, Fig. 2A). Dual luciferase reporter showed that miR-944 mimics only reduced the luciferase activity of wt-SNHG6, but had little effect on mut-SNHG6 in C666-1 cells (Fig. 2B). Additionally, SNHG6 was negatively correlated with miR-944 expression in NPC tissues (Fig. 2C). To further confirm their relationship, si-SNHG6, SNHG6 vector, miR-944 mimics or miR-944 inhibitor was transfected into C666-1 cells, respectively. We found that miR-944 expression was inhibited by SNHG6 vector and enhanced by si-SNHG6 in C666-1 cells (Fig. 2D). Meanwhile, miR-944 mimics reduced SNHG6 expression, while miR-944 inhibitor increased SNHG6 expression in C666-1 cells (Fig. 2E). These findings reveal that the tendency of mutual restrain existed between SNHG6 and miR-944 expression in NPC.

\section{SNHG6/miR-944 axis regulates NPC progression.}

Then, the regulatory mechanism of SNHG6/miR-944 was investigated in NPC cells. First, miR-944 inhibitor was transfected into C666-1 cells containing si-SNHG6. We found that SNHG6 expression was decreased by si-SNHG6. But miR-944 inhibitor restored this decreased expression of SNHG6 (Fig. 3A). In addition, knockdown of SNHG6 was found to restrain C666-1 cell proliferation. But miR-944 inhibitor abolished the inhibitory effect of si-SNHG6 on cell proliferation (Fig. 3B). Meanwhile, cell migration and invasion were also suppressed by knockdown of SNHG6. And the inhibition of cell migration and invasion was recovered by miR-944 inhibitor (Fig. 3C, 3D). To explore the role of miR-944 in NPC cells, miR-944 mimics and SNHG6 vector were transfected into C666-1 cells. MiR-944 expression was increased by its mimics. Upregulation of SNHG6 reduced the increased expression of miR-944 (Fig. 3E). Functionally, overexpression of miR-944 restrained cell proliferation, migration and invasion in C666-1 cells. Furthermore, SNHG6 vector weakened the inhibitory effect of miR-944 in Y79 cells (Fig. 3F, 3G, 3H). The results imply that SNHG6/miR-944 axis plays an important role in NPC progression.

\section{MiR-944 directly targets RGS17.}


In addition, RGS17 was predicted to be a potential target for miR-944 in TargetScan database (http://www.targetscan.org, Fig. 4A). Luciferase reporter assay showed that miR-944 mimics decreased the luciferase activity of wt-RGS17, indicating that miR-944 can bind with the 3'-UTR of RGS17 (Fig. 4B). Meanwhile, RGS17 expression was declined by miR-944 mimics and enhanced by miR-944 inhibitor in C666-1 cells (Fig. 4C). However, upregulation of SNHG6 increased RGS17 expression, whereas SNHG6 downregulation inhibited RGS17 expression in C666-1 cells (Fig. 4D). Besides that, upregulation of RGS17 was found in NPC tissues compared with normal tissues (Fig. 4E). Furthermore, a negative correlation between miR-944 and RGS17 expression was identified in NPC tissues (Fig. 4F). At the same time, SNHG6 was found to be positively correlated with RGS17 expression in NPC tissues (Fig. 4G). The results reveal that RGS17 may be involved in the regulation of SNHG6/miR-944 axis.

\section{SNHG6/miR-944 is involved in NPC tumorigenesis by regulating RGS17.}

To investigate the role of RGS17 in NPC as well as its interaction with SNHG6/miR-944, SNHG6 vector or miR-944 inhibitor was transfected into C666-1 cells containing si-RGS17. We found that the decreased expression of RGS17 induced by si-RGS17 was restored by SNHG6 vector or miR-944 inhibitor (Fig. 5A). Additionally, CCK-8 assay suggested that knockdown of RGS17 inhibited C666-1 cell proliferation. This inhibition of cell proliferation was recovered by SNHG6 upregulation or miR-944 downregulation (Fig. 5B). Meanwhile, cell migration and invasion were also suppressed by RGS17 downregulation. SNHG6 vector or miR-944 inhibitor also abolished the inhibitory effect of si-RGS17 on C666-1 cell migration and invasion (Fig. 5C, 5D). Collectively, SNHG6/miR-944 axis regulates NPC progression by regulating RGS17 expression.

\section{Discussion}

Recently, more and more studies have shown that IncRNA plays a regulatory role in various diseases and tumors. Here, the regulatory mechanism of IncRNA SNHG6 was investigated in NPC. In particular, upregulation of SNHG6 was found in NPC. Furthermore, the abnormal expression of SNHG6 was associated with TNM stage and distant metastasis in NPC patients. Functionally, knockdown of SNHG6 restrained cell viability and mobility in NPC. Similar to our results, SNHG6 expression was also increased in other malignancies, such as glioma and hepatocellular carcinoma $[19,20]$. In addition, high expression of SNHG6 was correlated with clinical stage and lymph node metastasis in patients with renal cell carcinoma [21], which is consistent with our results. The promoting effect of SNHG6 on cell proliferation, migration and invasion was also detected in colorectal cancer and breast cancer [22, 23]. The carcinogenesis of SNHG6 was also identified in NPC.

Previous studies have demonstrated that IncRNAs exert effect in cancer initiation and progression by acting as a ceRNA. LncRNA SNHG6 has been reported to function as a competing endogenous RNA by sponging miR-181a-5p in colorectal cancer [24]. In this study, SNHG6 also promoted NPC progression by sponging miR-944. In addition, downregulation of miR-944 was detected in NPC and associated with clinical stage and lymph node metastasis. Overexpression of miR-944 was found to suppress cell 
proliferation, migration and invasion in NPC. The downregulation and inhibitory role of miR-944 has been found in gastric cancer and lung adenocarcinoma $[25,26]$. These findings are similar to our results. Besides that, miR-944 has been reported to inhibit the malignancy of hepatocellular carcinoma by directly targeting IGF-1R [27]. In our research, RGS17 was confirmed to be a direct target of miR-944.

RGS17 has been found to be upregulated in lung cancer and prostate cancer $[28,29]$. Functionally, overexpression of RGS17 promoted cell proliferation, migration and invasion in hepatocellular carcinoma [30]. Here, RGS17 expression was also increased in NPC. Knockdown of RGS17 played an inhibitory effect in NPC, which is similar to the above results. Moreover, miR-944 blocked NPC progression by suppressing RGS17 expression. Consistent with our results, miR-203 has been proposed to restrain cell proliferation, invasion, and migration in non-small-cell lung cancer by downregulating RGS17 [31]. Linc00483 was found to accelerate tumorigenesis of cervical cancer by regulating miR-508-3p/RGS17 axis [32]. In present study, IncRNA SNHG6 was positively correlated with RGS17 expression in NPC. Upregulation of SNHG6 could weaken the inhibitory effect of RGS17 silencing in NPC. These results reveal that SNHG6 promoted NPC progression by upregulating RGS17.

\section{Conclusion}

In summary, SNHG6 is upregulated in NPC and serves as an oncogene. In particular, SNHG6 promotes cell proliferation, migration and invasion by competitively binding to miR-944 and upregulating RGS17. Our research will provide underlying therapeutically targets for the diagnosis and treatment of NPC.

\section{Declarations}

\section{Acknowledgements}

Not applicable

\section{Funding}

No funding was received

\section{Availability of data and materials}

The datasets used and/or analyzed during the current study are available from the corresponding author on reasonable request.

\section{Authors' contributions}

YH designed the study and drafted the manuscript. XL and GL were responsible for the collection and analysis of the experimental data. $\mathrm{XJ}$ and ZS revised the manuscript critically for important intellectual content. All authors read and approved the final manuscript. 


\section{Ethical approval}

The study was approved by Ethical Committee of Qilu Hospital of Shandong University (Qingdao) and conducted in accordance with the ethical standards.

\section{Consent for publication}

Not applicable.

\section{Competing interests}

The authors declare that they have no competing interests.

\section{References}

1. Yu MC, Yuan JM. Epidemiology of nasopharyngeal carcinoma. Semin Cancer Biol. 2002;12:421-9.

2. Tian YM, Guan Y, Xiao WW, Zeng L, Liu S, Lu TX, et al. Long-term survival and late complications in intensity-modulated radiotherapy of locally recurrent T1 to T2 nasopharyngeal carcinoma. Head Neck. 2016;38:225-31.

3. Lee AW, Ng WT, Chan YH, Sze H, Chan C, Lam TH. The battle against nasopharyngeal cancer. Radiother Oncol. 2012;104:272-8.

4. Beermann J, Piccoli MT, Viereck J, Thum T. Non-coding RNAs in Development and Disease: Background, Mechanisms, and Therapeutic Approaches. Physiol Rev. 2016;96:1297-325.

5. Ji Y, Wang M, Li X, Cui F. The Long Noncoding RNA NEAT1 Targets miR-34a-5p and Drives Nasopharyngeal Carcinoma Progression via Wnt/beta-Catenin Signaling. Yonsei Med J. 2019;60:336-45.

6. Zhang W, Du M, Wang T, Chen W, Wu J, Li Q, et al. Long non-coding RNA LINC01133 mediates nasopharyngeal carcinoma tumorigenesis by binding to YBX1. Am J Cancer Res. 2019;9:779-90.

7. Jafari-Oliayi A, Asadi MH. SNHG6 is upregulated in primary breast cancers and promotes cell cycle progression in breast cancer-derived cell lines. Cell Oncol (Dordr). 2019;42:211-21.

8. Li M, Bian Z, Yao S, Zhang J, Jin G, Wang X, et al. Up-regulated expression of SNHG6 predicts poor prognosis in colorectal cancer. Pathol Res Pract. 2018;214:784-9.

9. Cai G, Zhu Q, Yuan L, Lan Q. LncRNA SNHG6 acts as a prognostic factor to regulate cell proliferation in glioma through targeting p21. Biomed Pharmacother. 2018;102:452-7.

10. Zhang Y, Li R, Ding X, Zhang K, Qin W. Upregulation of long non-coding RNA SNHG6 promote esophageal squamous cell carcinoma cell malignancy and its diagnostic value. Am J Transl Res. 2019;11:1084-91.

11. Yan K, Tian J, Shi W, Xia H, Zhu Y. LncRNA SNHG6 is Associated with Poor Prognosis of Gastric Cancer and Promotes Cell Proliferation and EMT through Epigenetically Silencing p27 and Sponging miR-101-3p. Cell Physiol Biochem. 2017;42:999-1012. 
12. Wen L, Li Y, Jiang Z, Zhang Y, Yang B, Han F. miR-944 inhibits cell migration and invasion by targeting MACC1 in colorectal cancer. Oncol Rep. 2017;37:3415-22.

13. Liu M, Zhou K, Cao Y. MicroRNA-944 Affects Cell Growth by Targeting EPHA7 in Non-Small Cell Lung Cancer. Int J Mol Sci. 2016;17.

14. He Z, Xu H, Meng Y, Kuang Y. miR-944 acts as a prognostic marker and promotes the tumor progression in endometrial cancer. Biomed Pharmacother. 2017;88:902-10.

15. He H, Tian W, Chen $\mathrm{H}$, Jiang K. MiR-944 functions as a novel oncogene and regulates the chemoresistance in breast cancer. Tumour Biol. 2016;37:1599-607.

16. Xu M, Chen X, Lin K, Zeng K, Liu X, Xu X, et al. IncRNA SNHG6 regulates EZH2 expression by sponging miR-26a/b and miR-214 in colorectal cancer. J Hematol Oncol. 2019;12:3.

17. Li L, Luo HS. G-Protein Signaling Protein-17 (RGS17) Is Upregulated and Promotes Tumor Growth and Migration in Human Colorectal Carcinoma. Oncol Res. 2018;26:27-35.

18. Sun Y, Fang R, Li C, Li L, Li F, Ye X, et al. Hsa-mir-182 suppresses lung tumorigenesis through down regulation of RGS17 expression in vitro. Biochem Biophys Res Commun. 2010;396:501-7.

19. Meng Q, Yang BY, Liu B, Yang JX, Sun Y. Long non-coding RNA SNHG6 promotes glioma tumorigenesis by sponging miR-101-3p. Int J Biol Markers. 2018;33:148-55.

20. Birgani MT, Hajjari M, Shahrisa A, Khoshnevisan A, Shoja Z, Motahari P, et al. Long Non-Coding RNA SNHG6 as a Potential Biomarker for Hepatocellular Carcinoma. Pathol Oncol Res. 2018;24:329-37.

21. An HX, Xu B, Wang Q, Li YS, Shen LF, Li SG. Up-regulation of long non-coding RNA SNHG6 predicts poor prognosis in renal cell carcinoma. Eur Rev Med Pharmacol Sci. 2018;22:8624-9.

22. Wang X, Lai Q, He J, Li Q, Ding J, Lan Z, et al. LncRNA SNHG6 promotes proliferation, invasion and migration in colorectal cancer cells by activating TGF-beta/Smad signaling pathway via targeting UPF1 and inducing EMT via regulation of ZEB1. Int J Med Sci. 2019;16:51-9.

23. Lv P, Qiu X, Gu Y, Yang X, Xu X, Yang Y. Long non-coding RNA SNHG6 enhances cell proliferation, migration and invasion by regulating miR-26a-5p/MAPK6 in breast cancer. Biomed Pharmacother. 2019;110:294-301.

24. Yu C, Sun J, Leng X, Yang J. Long noncoding RNA SNHG6 functions as a competing endogenous RNA by sponging miR-181a-5p to regulate E2F5 expression in colorectal cancer. Cancer Manag Res. 2019;11:611-24.

25. Pan T, Chen W, Yuan X, Shen J, Qin C, Wang L. miR-944 inhibits metastasis of gastric cancer by preventing the epithelial-mesenchymal transition via MACC1/Met/AKT signaling. FEBS Open Bio. 2017;7:905-14.

26. An JC, Shi HB, Hao WB, Zhu K, Ma B. miR-944 inhibits lung adenocarcinoma tumorigenesis by targeting STAT1 interaction. Oncol Lett. 2019;17:3790-8.

27. Lv L, Wang X, Ma T. microRNA-944 inhibits the malignancy of hepatocellular carcinoma by directly targeting IGF-1R and deactivating the PI3K/Akt signaling pathway. Cancer Manag Res. 2019;11:2531-43. 
28. Su WZ, Ren LF. MiRNA-199 inhibits malignant progression of lung cancer through mediating RGS17. Eur Rev Med Pharmacol Sci. 2019;23:3390-400.

29. Zhang LS, Ma HG, Sun FH, Zhao WC, Li G. MiR-203 inhibits the malignant behavior of prostate cancer cells by targeting RGS17. Eur Rev Med Pharmacol Sci. 2019;23:5667-74.

30. Zhang W, Qian S, Yang G, Zhu L, Zhou B, Wang J, et al. MicroRNA-199 suppresses cell proliferation, migration and invasion by downregulating RGS17 in hepatocellular carcinoma. Gene. 2018;659:228.

31. Chi Y, Jin Q, Liu X, Xu L, He X, Shen Y, et al. miR-203 inhibits cell proliferation, invasion, and migration of non-small-cell lung cancer by downregulating RGS17. Cancer Sci. 2017;108:2366-72.

32. Hu P, Zhou G, Zhang X, Song G, Zhan L, Cao Y. Long non-coding RNA Linc00483 accelerated tumorigenesis of cervical cancer by regulating miR-508-3p/RGS17 axis. Life Sci. 2019;234:116789.

\section{Figures}


A
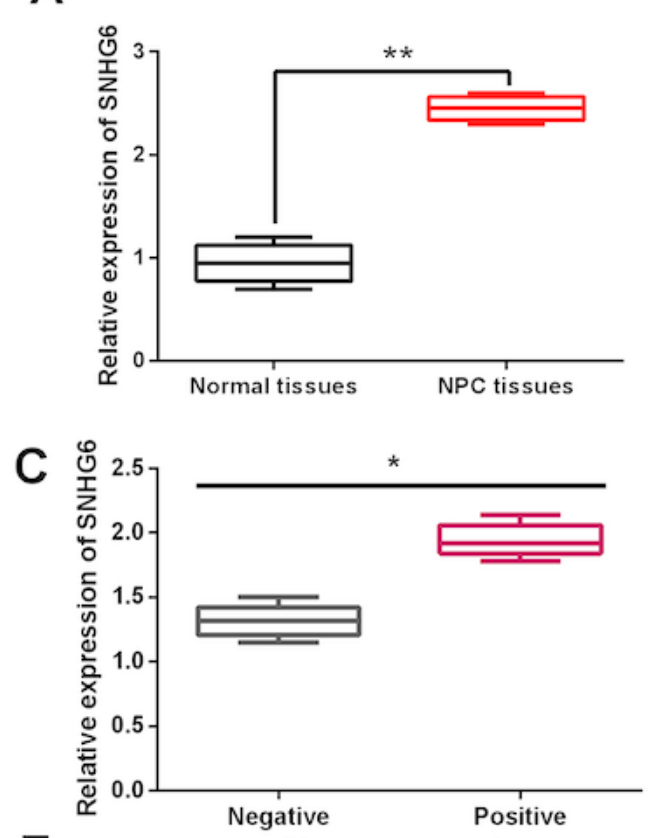

E

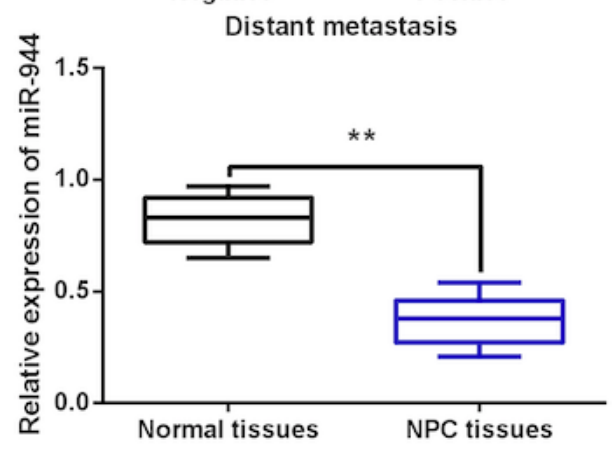

G

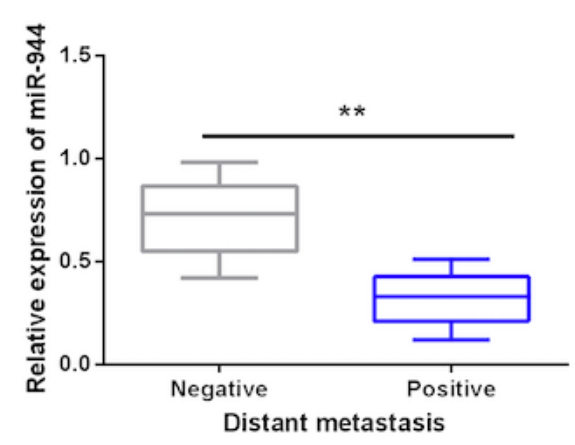

B

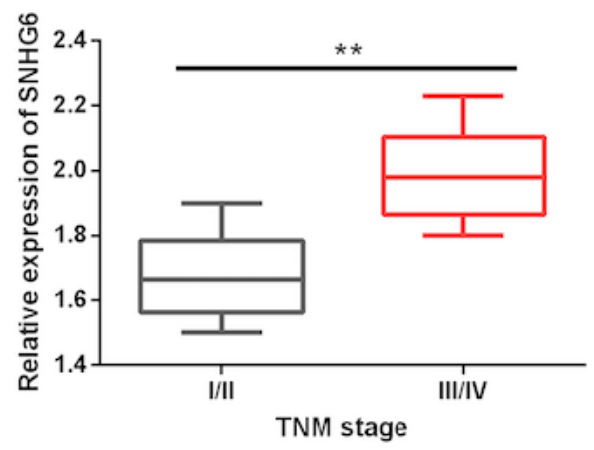

D

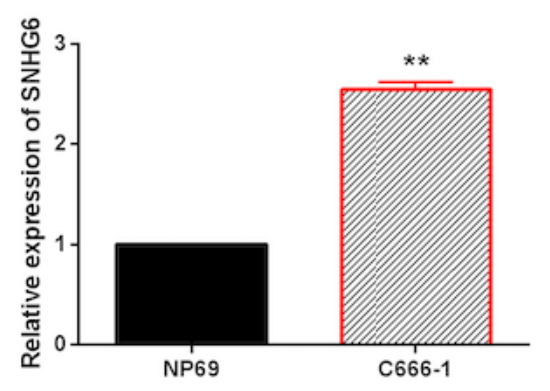

$\mathbf{F}$

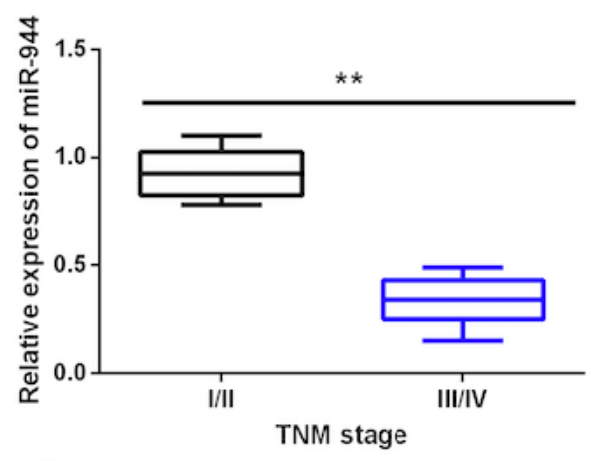

$\mathrm{H}$

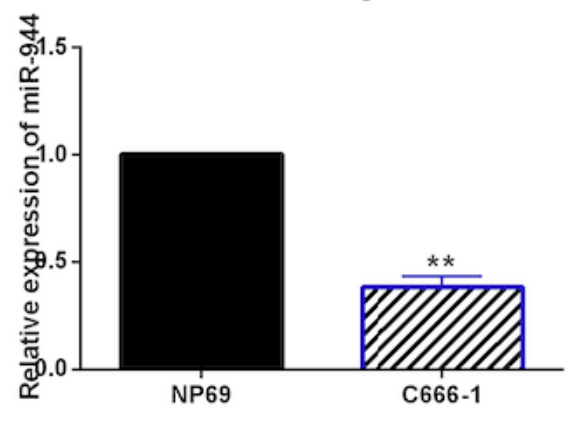

\section{Figure 1}

The abnormal expression of SNHG6 and miR-944 in NPC. (A) SNHG6 expression in NPC tissues and normal tissues (B) SNHG6 expression in stage III/IV and I/II tissues (C) SNHG6 expression in NPC tissues with or without distant metastasis (D) SNHG6 expression in NP69 and C666-1 cells (E) miR-944 expression in NPC tissues and normal tissues (F) miR-944 expression in stage III/IV and I/II tissues (G) 
miR-944 expression in NPC tissues with or without distant metastasis $(\mathrm{H})$ miR-944 expression in NP69 and $\mathrm{C} 666-1$ cells $* \mathrm{P}<0.01$

A

MiR-944

Wild of SNHG $63^{\prime}$-UTR

Mutant of SNHG 6 3'-UTR

B

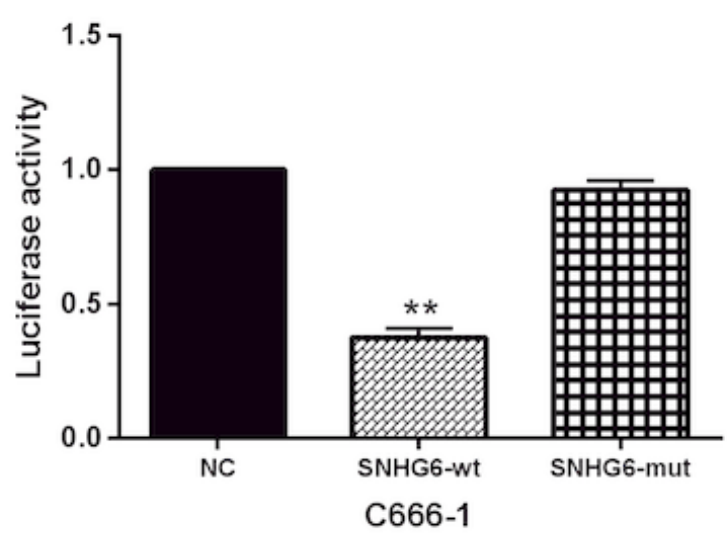

D

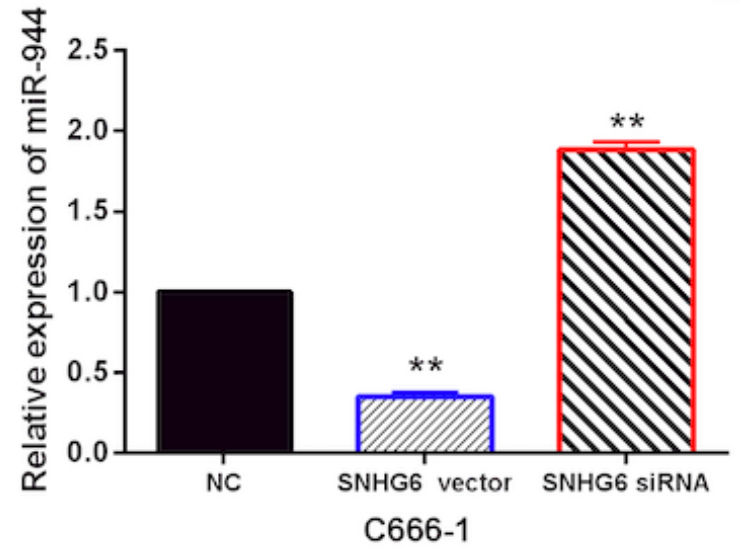

$E$
3'.......GAGUAGG CU CUACAU GUUAUUAAA....5'

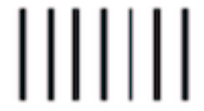

5'...GACAU GUAUGUAAGGCAAUAAU UU....3'

5'... GACAUGUAUGUAAGG GUUAUUAAU .....3'
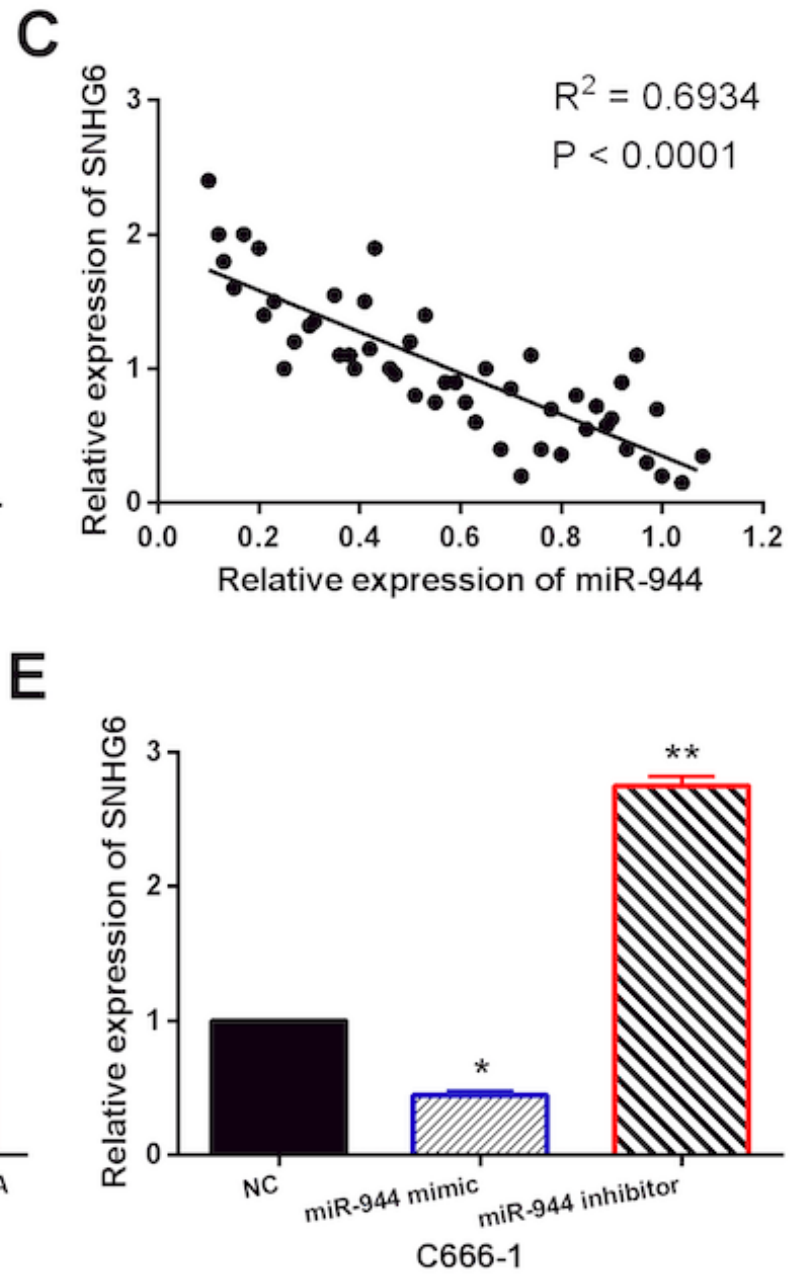

Figure 2

SNHG6 acts as a molecular sponge of miR-944. (A) The binding sites between SNHG6 with miR-944. (B) Luciferase reporter assay (C) MiR-944 was negatively correlated with SNHG6 in NPC tissues. (D) MiR-944 
expression in C666-1 cells with si-SNHG6 and SNHG6 vector (E) SNHG6 expression in C666-1 cells containing miR-944 mimics or inhibitor ** $\mathrm{P}<0.01$.

A

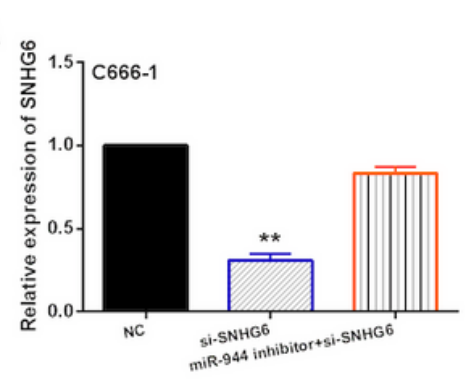

B
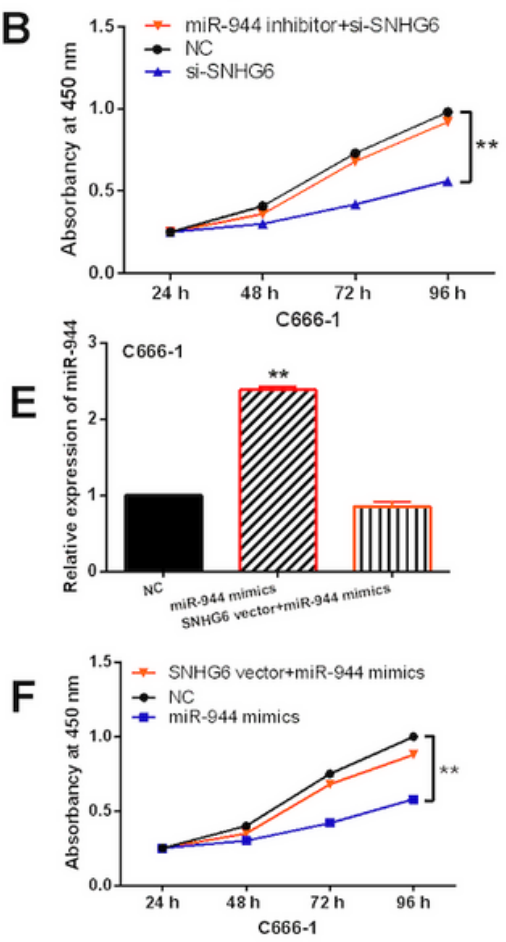

C
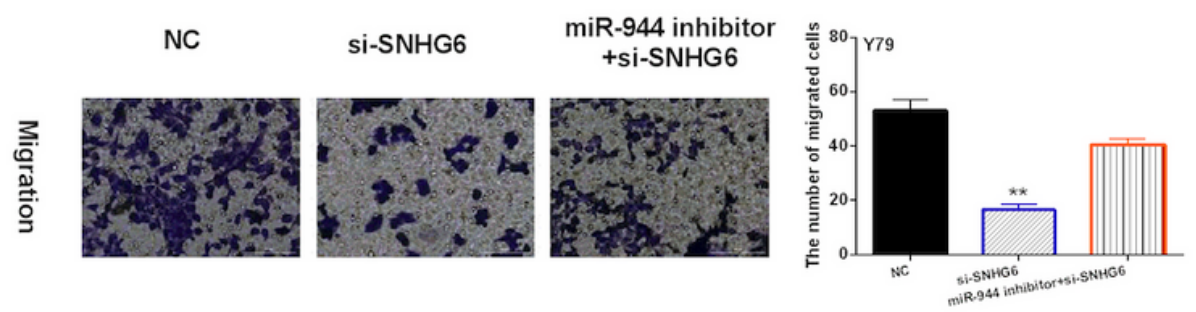

D $\quad$ si-SNHG $\quad$ miR-944 inhibitor
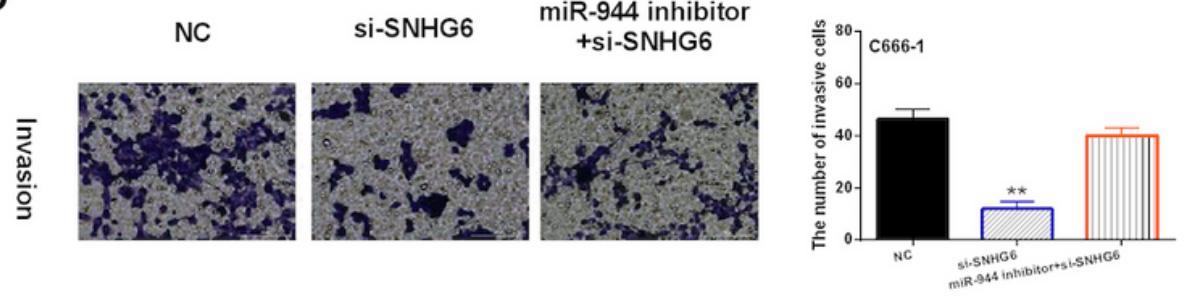

G

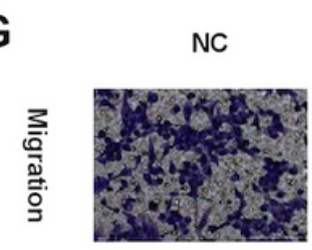

$\mathrm{H}$

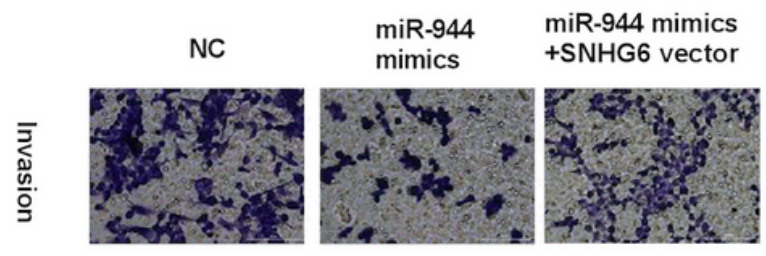

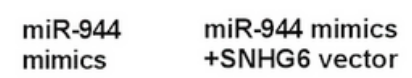
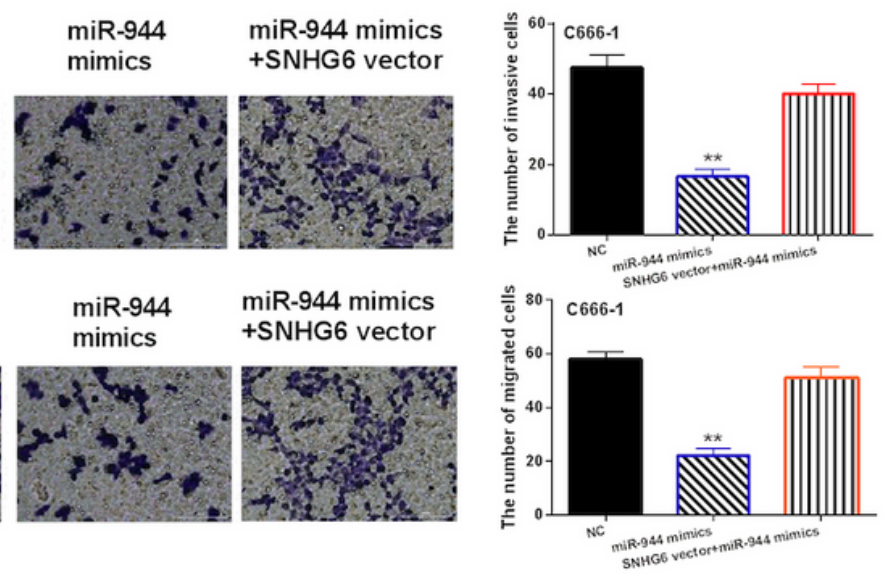

\section{Figure 3}

SNHG6/miR-944 axis regulates NPC progression. (A) SNHG6 expression in C666-1 cells with si-SNHG6 or si-SNHG6+miR-944 inhibitor. (B, C, D) Cell proliferation, migration and invasion in C666-1 cells with siSNHG6 or si-SNHG6+miR-944 inhibitor (E) MiR-944 expression in C666-1 cells with miR-944 mimics or miR-944 mimics+SNHG6 vector $(F, G, H)$ Cell proliferation, migration and invasion in C666-1 cells with miR-944 mimics or miR-944 mimics+SNHG6 vector ** $\mathrm{P}<0.01$ 
A

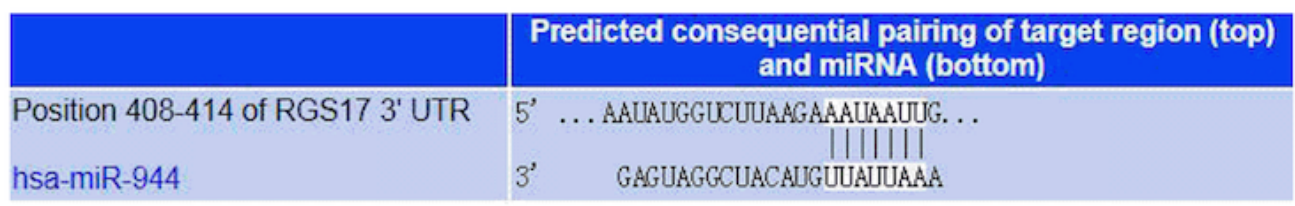

B

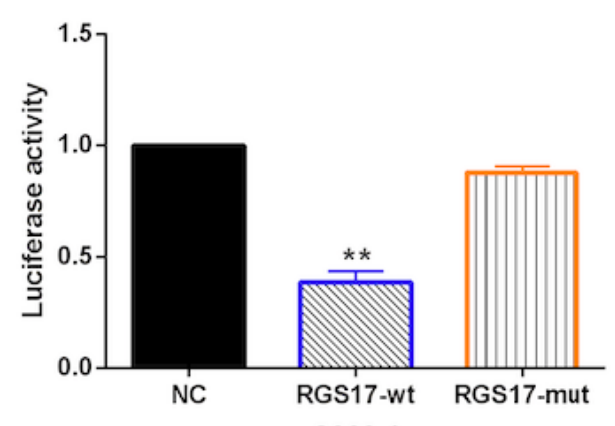

D

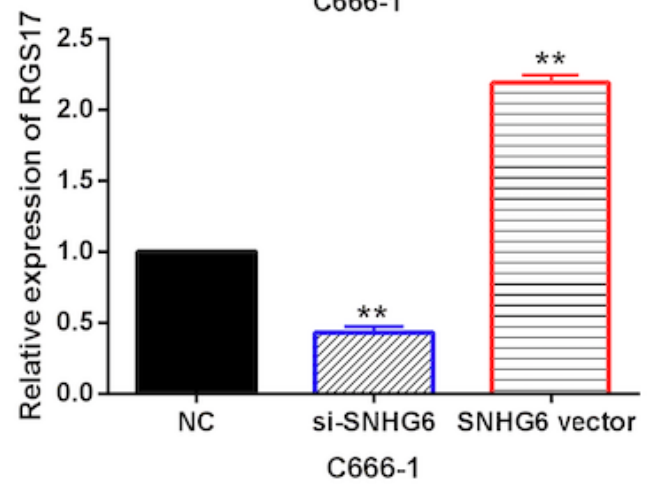

$\mathbf{F}$

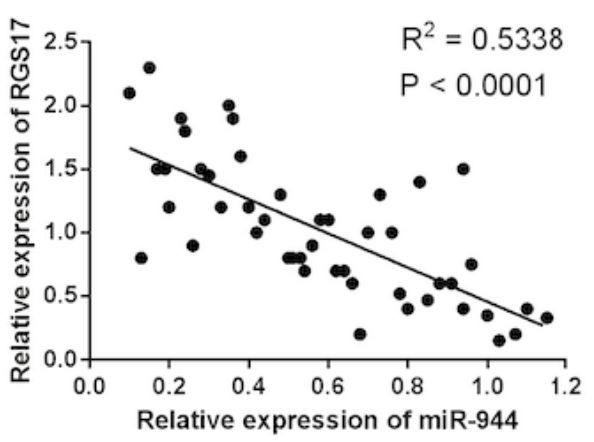

C

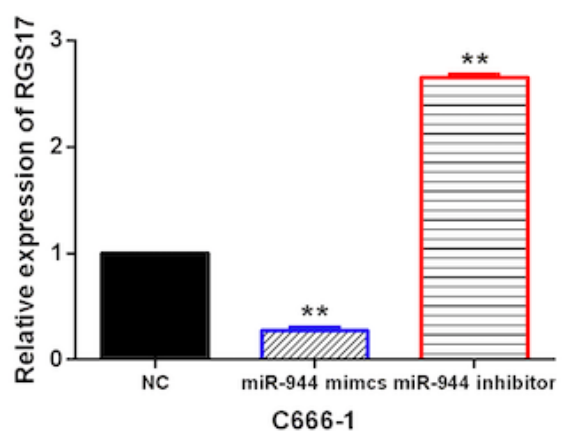

$\mathrm{E}$

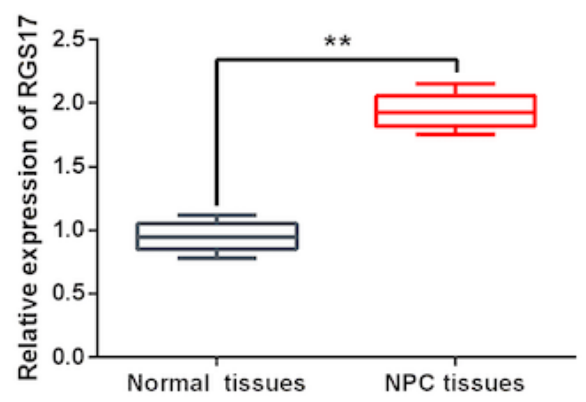

G

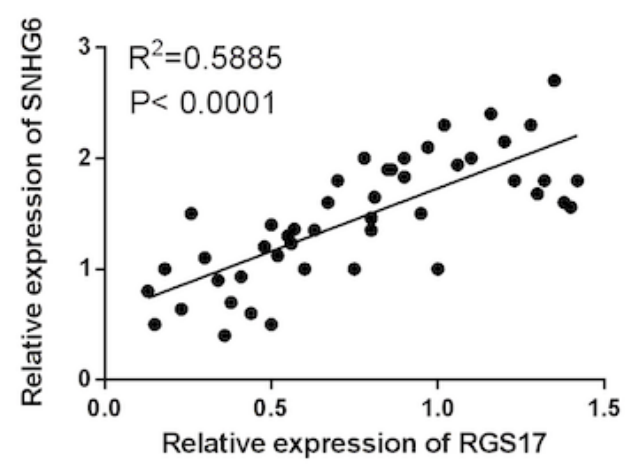

\section{Figure 4}

MiR-944 directly targets RGS17. (A) The binding site between RGS17 and miR-944. (B) Luciferase reporter assay (C) RGS17 expression regulated by miR-944 mimics or inhibitor in C666-1 cells (D) RGS17 expression regulated by si-SNHG6 or SNHG6 vector in C666-1 cells (E) RGS17 expression in NPC tissues and normal tissues (F) MiR-944 was negatively correlated with RGS17 in NPC tissues. (G) RGS17 was positively correlated with SNHG6 in NPC tissues. ${ }^{* *} \mathrm{P}<0.01$. 
A

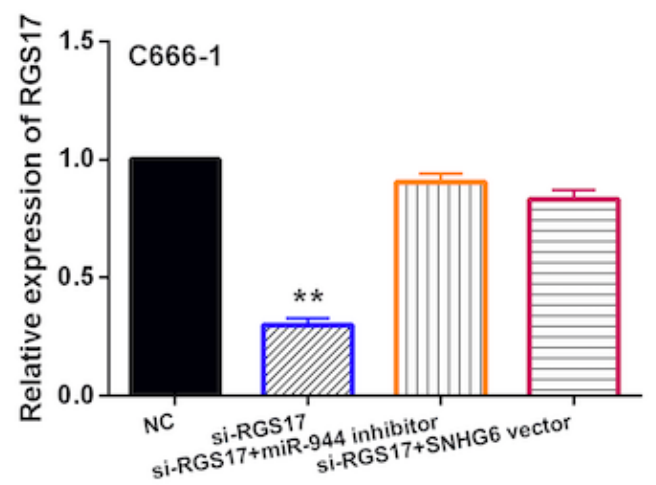

C

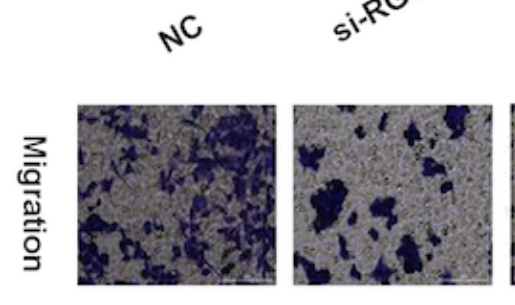

D
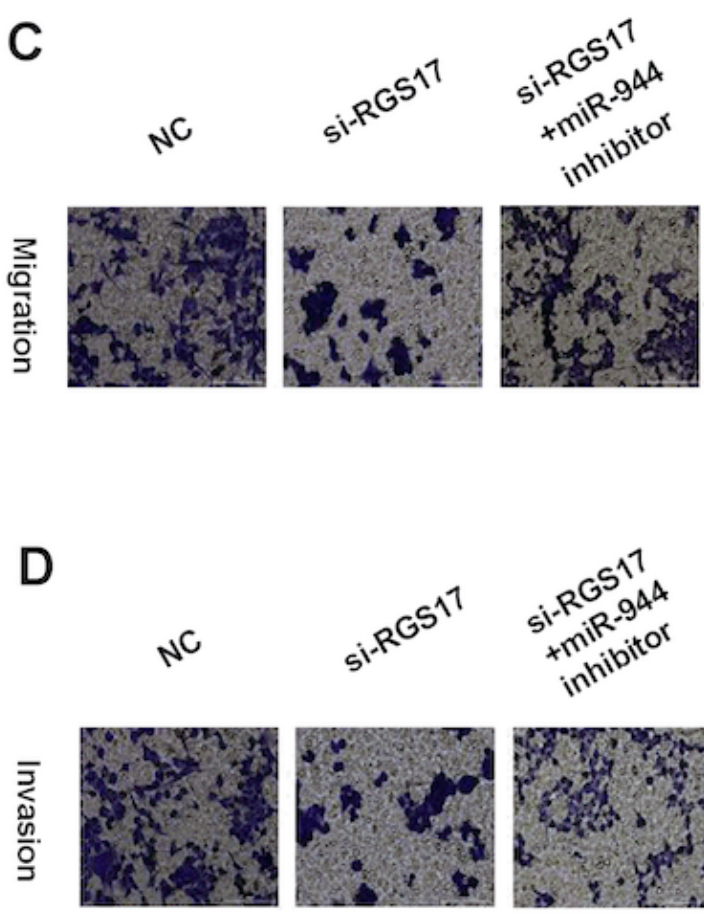
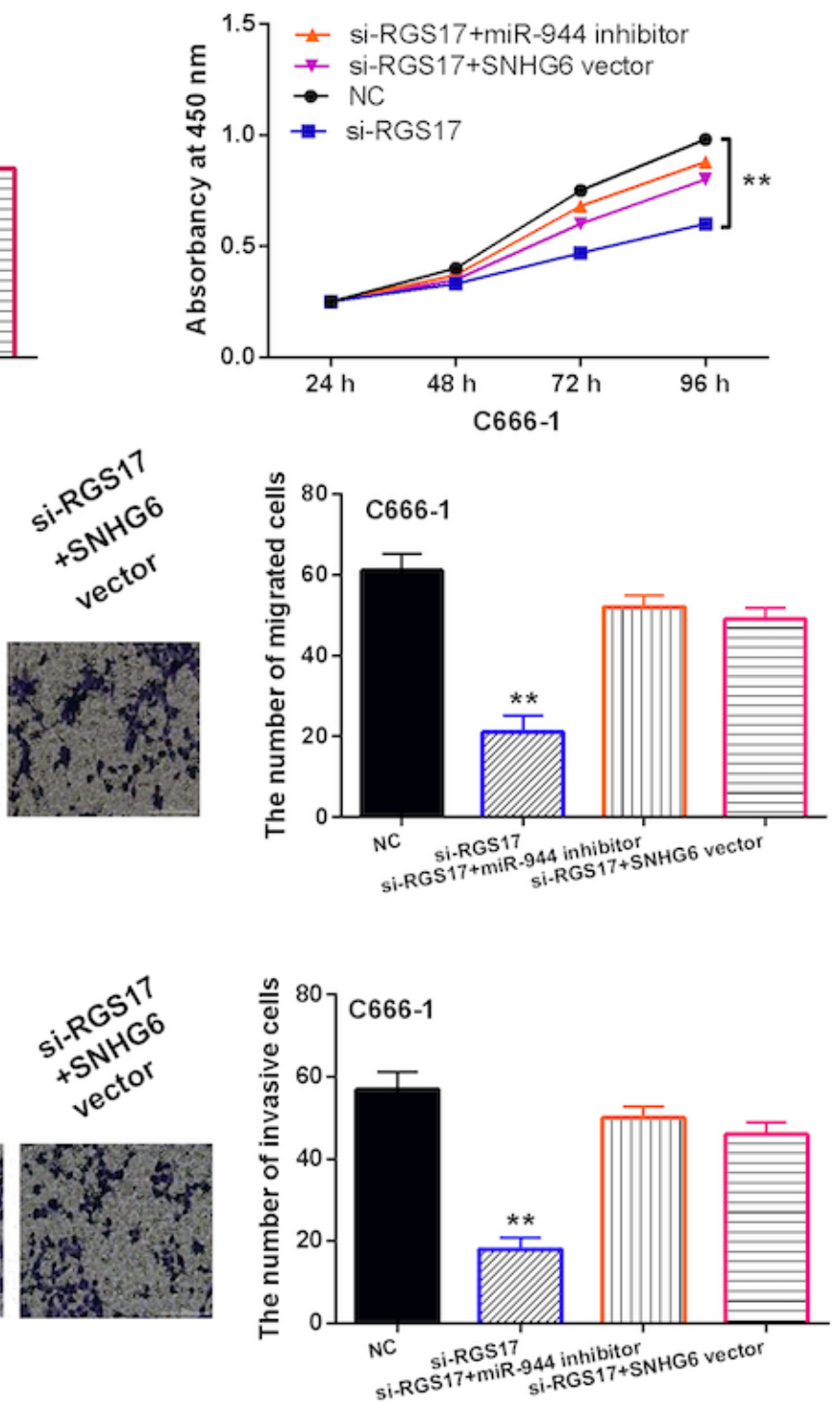

\section{Figure 5}

SNHG6/miR-944 is involved in NPC tumorigenesis by regulating RGS17. (A) RGS17 expression in C666-1 cells with si-RGS17, si-RGS17+miR-944 inhibitor or si-RGS17+SNHG6 vector. (B, C, D) Cell proliferation, migration and invasion in C666-1 cells with si-RGS17, si-RGS17+miR-944 inhibitor or si-RGS17+SNHG6 vector $* \star \mathrm{P}<0.01$ 